\title{
Large Eddy Simulation study of a fully developed thermal wind-turbine array boundary layer
}

Marc Calaf, Charles Meneveau and Marc Parlange

\section{Introduction}

When wind turbines are arranged in large wind farms, their efficiency decreases significantly due to wake effects and to complex turbulence interactions with the atmospheric boundary layer (ABL) [1]. For large wind farms whose length exceeds the ABL height by over an order of magnitude, a "fully developed" flow regime may be established $[1,2,3]$. In this asymptotic regime, the changes in the stream-wise direction are small compared to the more relevant vertical exchange mechanisms. Such a fully developed wind-turbine array boundary layer (WTABL) has recently been studied [2] using Large Eddy Simulations (LES) under neutral stability conditions. The simulations showed the existence of two log-laws, one above (characterized by: $\left.u_{*}^{h i}, z_{o}^{h i}\right)$ and one below $\left(u_{*}^{l o}, z_{o}^{l o}\right)$ the wind turbine region. This enabled the development of more accurate parameterizations of the effective roughness scale for a wind farm. Now, a suite of Large Eddy Simulations, in which wind turbines are modeled as in [2] using the classical drag disk concept are performed, again in neutral conditions but also considering temperature. Figure 1 shows a schematic of the geometry of the simulation.

The aim is to study the effects of different thermal ABL stratifications, and thus to study the efficiency and characteristics of large wind farms and the associated land-atmosphere interactions for realistic atmospheric flow regimes. Such studies

\footnotetext{
Marc Calaf

Department of Environmental Engineering, Ecole Polythecnique Fédérale de Lausanne (EPFL), GR A0 445, Station 2, 1015 Lausanne, Switzerland, e-mail: marc.calaf@epfl.ch

Charles Meneveau

Department of Mechanical Engineering, and Center for Environmental and Applied Fluid Mechanics, Johns Hopkins University, 3400 North Charles Street, Baltimore MD 21218, USA, e-mail: meneveau@jhu.edu

Marc Parlange

Department of Environmental Engineering, Ecole Polythecnique Fédérale de Lausanne (EPFL), GR A0 412, Station 2, 1015 Lausanne, Switzerland, e-mail: marc.parlange@epfl.ch
} 
help to unravel the physics involved in extensive aggregations of wind turbines, allowing us to design better wind farm arrangements. As a first step, temperature is treated in a passive mode, allowing us to focus the study on the influence of a large WFABL into the scalar fluxes. By considering various turbine loading factors, surface roughness values and different atmospheric stratifications, it is possible to analyze the influence of these parameters on the induced surface roughness, and the sensible heat roughness length. These last two parameters can be used to model wind turbine arrays in simulations of atmospheric dynamics at larger (regional and global) scales [4], where the coarse meshes used do not allow to account for the specifics of each wind turbine. Results from different sets of simulations are presented, for which also the corresponding effective roughness length-scales can be determined. The results also help our understanding of how wind turbines affect scalar transport processes in the turbine wakes.

By using a simple drag disk approach for modeling the wind turbines, it is found that the surface heat flux inside the thermal wind-turbine array boundary layer is increased. This is the result of two competing effects: (1) a major increase on $u_{*, h i}$; (2) a smaller decrease due to lower $u_{*, l o}$ near the ground.

\section{Description of numerical method}

In this work we consider flow that is neutrally stratified, and driven by an imposed pressure gradient with temperature as a passive scalar.

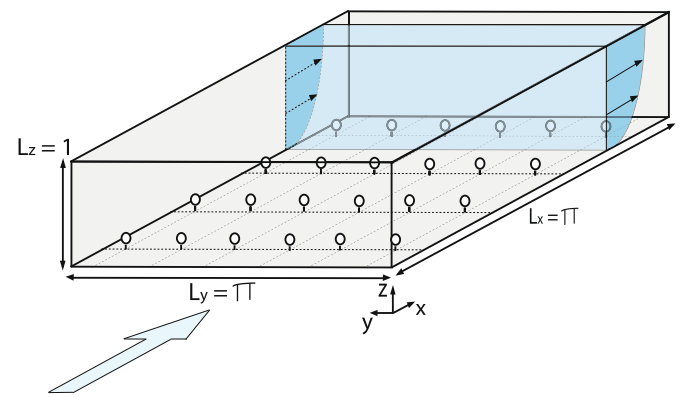

Fig. 1 Schematic sketch of the modeled wind farm, with dimensions: $\left(L_{x}, L_{y}, L_{z}\right)=(\pi, \pi, 1) \times 1000 \mathrm{~m}$ and $128^{3}$ grid cells. With the periodic boundary conditions the simulations reproduce the conditions of a fully developed Wind Turbine Array Boundary Layer (WFABL). 
Therefore, the LES is based on the filtered incompressible Navier-Stokes equations, the continuity equation and an equation for potential temperature, i.e.

$$
\begin{gathered}
\partial_{i} \widetilde{u}_{i}=0 \\
\partial_{t} \widetilde{u}_{i}+\partial_{j}\left(\widetilde{u}_{i} \widetilde{u}_{j}\right)=-\partial_{i} \widetilde{p}^{*}-\partial_{j} \tau_{i j}+f_{i}-\delta_{i 1} \partial_{1} p_{\infty} / \rho \\
\partial_{t} \widetilde{\theta}+\widetilde{u}_{j} \partial_{j} \widetilde{\theta}=-\partial_{j} \pi_{j}
\end{gathered}
$$

where $\widetilde{u}_{i}$ is the filtered velocity field, $\widetilde{\theta}$ is the filtered temperature field, and $\widetilde{p}^{*}$ is the filtered modified pressure equal to $\widetilde{p} / \rho+\tau_{k k} / 3-p_{\infty} / \rho$. Further, $\tau_{i j}$ is the subgridscale stress term. Its deviatoric part $\left(\tau_{i j}-\delta_{i j} \tau_{k k} / 3\right)$ is modeled using an eddy viscosity subgrid-scale model, as discussed further below; the trace of this term $\left(\tau_{k k} / 3\right)$ is combined into the modified pressure, as is common practice in incompressible LES. Equivalently, $\pi_{j}$ is the scalar SGS flux term. (Note that if temperature was included as an active scalar, an extra term would be added on the right hand side of the momentum equation. Using Boussinesq's approximation this would be given by: $\left.g \frac{\tilde{\theta}-\langle\widetilde{\theta}\rangle}{\langle\tilde{\theta}\rangle}\right)$. Figure 1 shows a sketch of the computational domain with representative dimensions: $\left(L_{x}, L_{y}, L_{z}\right)=(\pi, \pi, 1) \times 1000 \mathrm{~m}$ and $128^{3}$ grid cells.

The force $f_{i}$ is added for modeling the effects of the wind turbines in the momentum equation using the "drag disk" approach in LES [5], with a new local variant $[2,6]$. Since simulations are done at very large Reynolds numbers and the bottom surface as well as the wind-turbine effects are parameterized, viscous stresses are neglected. In the real case of wind turbines in the atmospheric boundary layer, the flow is forced by geostrophic wind and in the outer layer is affected by Coriolis accelerations. The flow changes direction near the ground, and for a given geostrophic wind direction, the turning depends upon the shear stresses (momentum exchanges) at the bottom surface. Since these are not known ahead of time, and we wish to have a mean wind that is perpendicular to the wind-turbine disks in the array to be simulated, in the simulations we prefer to use forcing with an imposed pressure gradient $\partial_{1} p_{\infty}$ in the $x_{1}$ direction. The results of the simulations, especially in the surface layer region, can still be interpreted in the context of geostrophic wind forcing.

The skew-symmetric form of the NS equation is implemented. The numerical discretization follows the approach used by Moeng [7] and Albertson \& Parlange [8], which combines a pseudo-spectral discretization in the horizontal directions and a centered second-order finite differencing in the vertical direction. With the periodic boundary conditions the simulations reproduce the conditions of a fully developed Wind Turbine Array Boundary Layer (WFABL). A second order accurate Adams-Bashforth scheme is used for time integration. The subgrid model used is the dynamic Smagorinsky model [9] using the Lagrangian scale-dependent version as described in [10]. The nonlinear convective terms and the SGS stress are dealiased using the $3 / 2$ rule.

In the streamwise direction, we use fully periodic boundary conditions (in accordance with the spectral discretization). The top boundary uses zero vertical velocity and zero shear stress boundary condition (same for temperature). At the bottom sur- 
face, we use a classic imposed wall stress boundary condition relating the wall stress to the velocity at the first grid-point and the surface heat flux to the temperature at the first grid-point using the standard log (Monin-Obukhov) similarity law [4].

$$
\begin{gathered}
\tau_{w 1}=-\left(\frac{\kappa}{\ln z / z_{0, l o}}\right)^{2}\left(\widehat{\widetilde{u}}^{2}+\widehat{\widetilde{v}}^{2}\right)^{0.5} \widehat{\widetilde{u}} \\
\tau_{\theta w}=\frac{\left(\theta_{s}-\tilde{\theta}\right) u_{*} \kappa}{\ln \left(z / z_{0, l o}\right)}
\end{gathered}
$$

where the hat on $\widehat{\widetilde{u}}$ and $\widehat{\widetilde{v}}$ represents a local average obtained by filtering the LES velocity field with filter width $2 \Delta$ (see [9] for more details about such filtering).

\section{LES results}

Figure 2 shows vertical profiles of the horizontally averaged heat fluxes. Lines with symbols represent the case with no wind turbines. Lines without symbols are the reference case without wind turbines. The dot-dashed lines represent the subgrid-scale component of the heat flux, which for both cases, with and without wind turbines, are very similar. The dashed lines account for the turbulent Reynolds heat flux components, these being $\sim 15 \%$ larger near the ground (between 0 to $\sim 0.1 / H$ height) for the case with wind turbines. Such differences can also be observed in the dispersive (canopy) heat flux term, but at a height above the actual wind turbines (between $\sim 0.2 / H$ to $\sim 0.7 / H$ height). Overall, an increase of about $\sim 15 \%$ on the total heat flux (solid lines) is observed in the scenario where the wind turbines are present. This increase in the heat flux is the result of two competing effects: one, a major increase of $u_{*, h i}(\sim 50 \%)$; two, a decrease due to lower $u_{*, l o}$ near the ground. The increase of $u_{*, h i}$ is due to the increase in turbulence and mixing induced by the wind turbines blades; while the reduction of $u_{*, l o}$ is explained due to the slow down of the mean flow near the ground because of the wind turbines presence. For further details see [2].

Figure 3 compares vertical profiles between cases with different thrust coefficients $C_{t}^{\prime}=\{0,0.6,1.33,2\}$ of the horizontally averaged heat flux. The solid line represents the case where there are no wind turbines. $C_{t}^{\prime}=2$, represents the corresponding Betz limit case, while $C_{t}^{\prime}=1.33$ is closer to a real scenario. $C_{t}^{\prime}=0.6$ represents a weakly loaded case. Results show that the increase of the heat flux is proportional to the thrust coefficient, although this proportionality seems to reach an asymptote for the more loaded cases. 


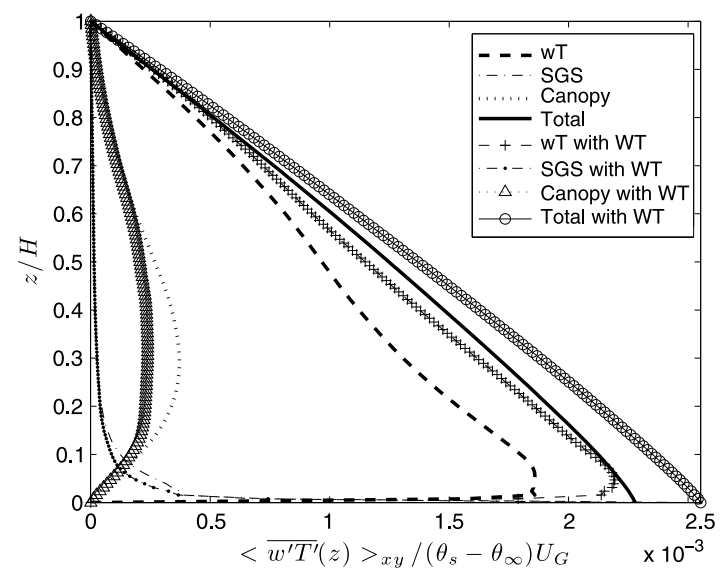

Fig. 2 Vertical profile of the spatially averaged heat flux, $\left\langle\overline{w^{\prime} \theta^{\prime}}\right\rangle_{x y}$. Symbols show the case with wind turbines. The dashed line shows the Reynolds surface heat flux, the dot-dashed line represents the subgrid-scale heat fluxes and the dotted line represents the diffusive (canopy) heat fluxes. The solid line is the total heat flux, resultant of adding all the three different components. These are normalized with the temperature difference between the surface and the top of the boundary layer times the geostrophic wind.

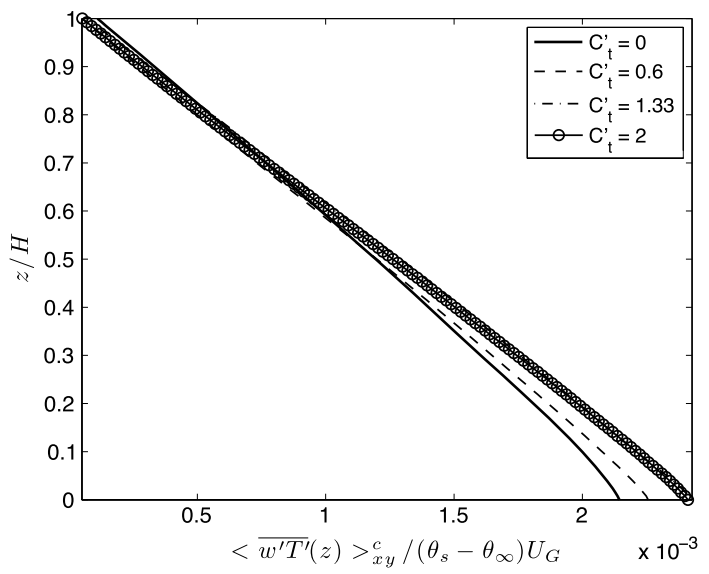

Fig. 3 Heat flux, vertical profiles. Comparison between cases with different thrust coefficients $\left(C_{t}^{\prime}\right)$. Solid line represents the case where there are no wind turbines $\left(C_{t}^{\prime}=0\right)$. Dashed line shows the case with the lightest thrust coefficient $\left(C_{t}^{\prime}=0.6\right)$. The dot-dashed line represents the case with an intermediate loading $\left(C_{t}^{\prime}=1.33\right)$, and the hollow circle case, represents the case corresponding to the Betz limit $\left(C_{t}^{\prime}=2\right)$. 


\section{Conclusions}

Results have shown that large wind farms do increase scalar fluxes, as it was earlier foreseen by [11]. This increase is the result of two competing effects: one, a major increase of $u_{*, h i}(\sim 50 \%)$; two, a decrease due to lower $u_{*, l o}$ near the ground. Also, it has been shown that higher thrust coefficients $\left(C_{t}^{\prime}\right)$ have an increasing effect on the scalar fluxes up to a certain asymptote. This asymptote should be analyzed with more detail. Therefore, we plan on studying different intermediate $C_{t}^{\prime}$ values among the ones tested by now, different surface roughnesses $\left(z_{0}\right)$, and wind turbine spacings $\left(S_{x}, S_{y}\right)$. This will also allow us to better asses the inter-relationship between the increase of scalar fluxes and these relevant wind farm parameters.

Acknowledgements M.C. was supported by (Swiss) SNF 200021-107910/1 Land-atmosphere interaction over complex terrain: large eddy simulation and field experiments. C.M. acknowledges funding from the US National Science Foundation (Projects CBET 0730922 and AGS 1045189).

\section{References}

1. Frandsen S., Barthelmie R., Pryor R., Rathmann O., Larsen S., Hojstrup J., Thogersen M.: Analytical modelling of wind speed deficit in large offshore wind farms. Wind. Energy $\mathbf{9}$ : 39-53, (2006).

2. Calaf M., Meneveau C., Meyers J.: Large Eddy Simulation study of fully developed windturbine array boundary layers. Phys. Fluids 22, 015110, (2010) doi:10.1063/1.3291077.

3. Cal R.B., Lebrón J., Kang H.S., Castillo L., Meneveau C.: Experimental study of the horizontally averaged flow structure in a model wind-turbine array boundary layer. J. Renewable Sustainable Energy 2, 013106, (2010) doi:10.1063/1.3289735.

4. Barrie D., Kirk-Davidoff D.: Weather response to Management of large wind turbine array. Atmos. Chem. Phys. Discuss. 9, 2917, (2009).

5. Jimenez A., Crespo A., Migoya E., Garcia J.: Advances in large-eddy simulation of a wind turbine wake. J. of Physics: Conference Series 75, 012041, (2007).

6. Meyers J., Meneveau C.: Large eddy simulations of large wind-turbine arrays in the atmospheric boundary layer. In Proceedings of the 48th AIAA Aerospace Sciences Meeting, January, Orlando (FL). AIAA paper 2010-827 (2010)

7. Moeng C.-H.: A large-eddy simulation model for the study of planetary boundary-layer turbulence. J. Atmos. Sci. 6:2311-2330, (1984).

8. Albertson J.D., Parlange M.B.: Surface length-scales and shear stress: implications for landatmosphere interaction over complex terrain. Water Resour. Res. 35:2121-2132, (1999a).

9. Germano M., Piomelli U., Moin P., Cabot W.H.: A dynamic subgrid-scale eddy viscosity model. Phys. Fluids A 3, 1760, (1991).

10. Bou-Zeid E., Meneveau C., Parlange M.B.: A scale dependent Lagrangian dynamic model for large eddy simulation of complex turbulent flows. Physics of Fluids 17, 025105, (2005).

11. Baidya-Roy S., Pacala S.W., Walko R.L.: Can large wind farms effect local meteorology? Journal of Geophysical Research 109, (2004). 